\title{
UTILITY OF NON-EFFORT DEPENDENT PULMONARY FUNCTION TESTS (NEDPFT) IN PEDIATRIC EMERGENCY DEPARTMENT (ED) PATIENTS WITH ACUTE ASTHMA EXACERBATION
}

\author{
K. Giordano ${ }^{1}$, M.E. Rodriguez ${ }^{2}$, M. Armani ${ }^{2}$, N. Green ${ }^{1}$, J. Richards ${ }^{1}$, T. Shaffer ${ }^{2}$, M.W. Attia ${ }^{1}$ \\ ${ }^{I}$ Pediatrics, ${ }^{2}$ Lung Research Center - Biomedical Research, Alfred I duPont Hospital, Wilmington, DE, USA
}

Background: Asthma represents a significant percentage of ED visits. Management decisions are based on clinical evaluation. Spirometry and peak flow have been used to monitor control but both are limited by being effort dependent. NEDPFT such as inductance plethysmography and respiratory profile monitor role have not been fully evaluated in ED setting.

Objective: To assess feasibility and objectivity of NEDPFT in disposition of asthmatic children

Methods: A prospective study of ED patients with acute asthma exacerbation ages 3 years to 18 years. NEDPFT were performed before and after therapy. Therapy was at the discretion of the treating physician. The main outcome measure is admission versus discharge. A comparison between standard measurements; vital signs and clinical asthma score and those obtained from the NEDPFT to determine baseline measurements and values associated with admission.

Results: Forty patients have been analyzed, of which 14 (35\%) were admitted, mean age of 7 years (SD +/4.5). No difference was noted in patient demographics, the initial heart rate or the respiratory rate $(\mathrm{p}>0.05)$. Based on normative standards ranges from the NEDPFT were analyzed; Higher phase angles and labored breathing indexes as well as clinical asthma score were found to be associated with admission $($ all $<0.05)$.

Conclusions: NEDPFT can give an objective value that can be used in the evaluation of an acute asthma exacerbation in the ED setting. Our data shows that the measurements are feasible in the age group enrolled and are indicative of the need for admission. 